\title{
Heating Detection and Temperature Control Method of Power Lithium Battery in Pure Electric Vehicle
}

\author{
Guanqiang Ruan, Changqing Yu, Xing Hu, Jing Hua \\ Mechanical Engineering School, Shanghai Dian Ji University, Shang Hai, China
}

\begin{abstract}
For the power battery of electric vehicles, especially pure electric vehicles, there is no perfect and comprehensive detection system and management system in the production and use links. By analyzing the working principle, structure and electrochemical characteristics of lithium-ion battery, the heat generation mechanism of lithiumion battery was studied. In this paper, the factors that affect the temperature characteristics of Li ion battery are described, and the corresponding relationship between the temperature rise of the battery and the ambient temperature is established. At the same time, the optimal temperature range of the battery pack discharge efficiency is determined. In this paper, the thermal effect model and heat generation rate model of lithium-ion battery are established, and then the thermal conductivity, specific heat capacity, density and other parameters of the thermal model are calculated. Finally, the initial and boundary conditions of the thermal model are determined, the simulation of heat generation temperature field is realized, and the temperature distribution of the battery after heat generation is obtained. In this paper, the flow mode of air is analyzed, and the fluid structure coupling model of battery air is established. Finally, the thermal field of the battery pack is simulated by setting the solver mode and boundary conditions, which makes a theoretical analysis for the preliminary design of the temperature control battery box. The test results show that the method proposed in this paper can meet the technical requirements of power lithium battery heating management of pure electric vehicles..
\end{abstract}

Keywords: Electric vehicle, lithium battery, heat detection, temperature control

\section{Introduction}

The development of society is inseparable from the supply of energy, and with the gradual development of society, human demand for energy is more and more huge, the development of new energy has become the only way out for all countries [1]. On the one hand, it is because there are fewer and fewer non renewable energy sources such as oil, which is not enough for the demand for development. Since the first industrial revolution, the demand for energy has been growing. However, non renewable resources such as oil are limited and will be used up one day. How will our future generations develop? [2-4] And many countries have suffered a lot of disasters and wars because of competing for energy. Therefore, it is imperative to develop new energy to replace non renewable energy. On the other hand, the pollution caused by fossil fuels is immeasurable, which will seriously affect the living environment of our future generations [5]. From the perspective of the long-term development of the automotive industry in the future, new energy vehicles are our only choice and the right choice.

Whether in China or abroad, new energy vehicles have become a trend in the development of the automotive industry, and the most typical representative is pure electric vehicles. From the beginning of the 21 st century, China has given great impetus to the development of pure electric vehicles, and some aspects have played a leading role [6]. Of course, the automobile manufacturers and government departments of other countries have also played a very positive role in the development of pure electric vehicles. We should work together to develop pure electric vehicles better. Pure electric vehicles from the beginning of the designer's careful design, to repeated laboratory tests, and then to small-scale preliminary production, until today's large-scale popularization, the development of pure electric vehicles from emerging industries to a new big market development stage. I believe that in the near future, electric vehicles will replace traditional fuel vehicles and change people's lives [7-9]. Under the background

ISSN: 0010-8189

www.converter-magazine.info 
of shortage of non renewable resources and serious environmental pollution, the emergence of new energy electric vehicles is inevitable. It will reduce human dependence on non renewable resources and reduce the environmental pollution caused by traditional oil vehicles, which has very important practical significance.

\section{Structure and working principle of lithium ion battery}

Lithium ion battery is a kind of rechargeable battery, which mainly depends on the lithium ion in the battery to do the back and forth movement between the positive and negative poles to charge and discharge.
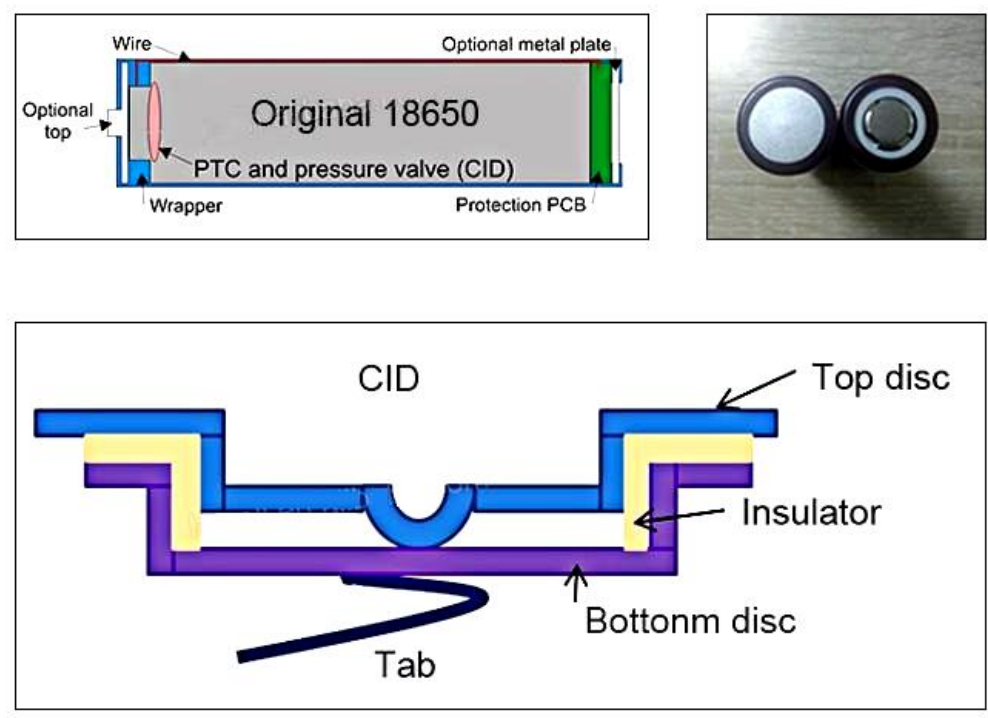

Fig 1: Structure of lithium ion battery

The lithium ion battery is mainly composed of five parts (as shown in Figure 1), which are electrode lead, positive electrode material, electrolyte, separator and negative electrode material.

Transition metal oxides are generally selected as cathode materials, and the common materials are $\mathrm{LiFePO}_{4}$, $\mathrm{LiNiO}_{2}, \mathrm{LiCoO}_{2}$ and $\mathrm{LiMn}_{2} \mathrm{O}_{4}$. In fact, the capacity of lithium-ion batteries has a great relationship with the electrochemical performance of cathode materials of lithium-ion batteries. Negative electrode materials affect the capacity of lithium ion batteries by affecting the insertion and extraction of lithium ions.

The main function of electrolyte is to transport the charge between positive and negative electrodes. It has high ionic conductivity. Some electrolytes can improve the activity of electrolyte in charge discharge chemical reaction, while others can not. Choosing the right electrolyte can not only improve the energy stored in the battery during charging and discharging, but also improve the power density and energy density of the battery, so that the battery can achieve the maximum efficiency [10].

The electrons inside the battery are likely to have a short circuit of the battery under the action of the current, so something is needed to separate the positive and negative poles. Therefore, the diaphragm plays such a role. It is located between the positive and negative electrodes, and its main function is to prevent short circuit. It can also make lithium ion through electrolyte to have electrochemical reaction between the positive and negative plates without barrier, and block the electrons inside the battery from passing through the diaphragm.

The cathode material of lithium-ion battery is a compound containing lithium, only lithium ion exists but no lithium metal exists, and the anode material is carbon material. As shown in Figure 2, when the lithium-ion battery is being charged, the lithium ion is de embedded from the Yellow positive electrode on the left, enters the blue negative electrode through the middle electrolyte and separator, and then embedded into the negative electrode. 
It can be seen from the figure that the red electrons also flow from the positive electrode to the negative electrode. When the lithium-ion battery is discharging, the lithium ion will come out from the blue negative pole on the right, enter the Yellow positive pole through the electrolyte and separator in the middle, and then insert into the positive pole. It can be seen from the figure that the red electrons also flow from the negative pole to the positive pole. In the working process of lithium-ion battery charging and discharging, lithium ion between the positive and negative poles, successively through the middle of the electrolyte and separator, doing a round-trip movement, so we call the lithium-ion battery "rocking chair battery".

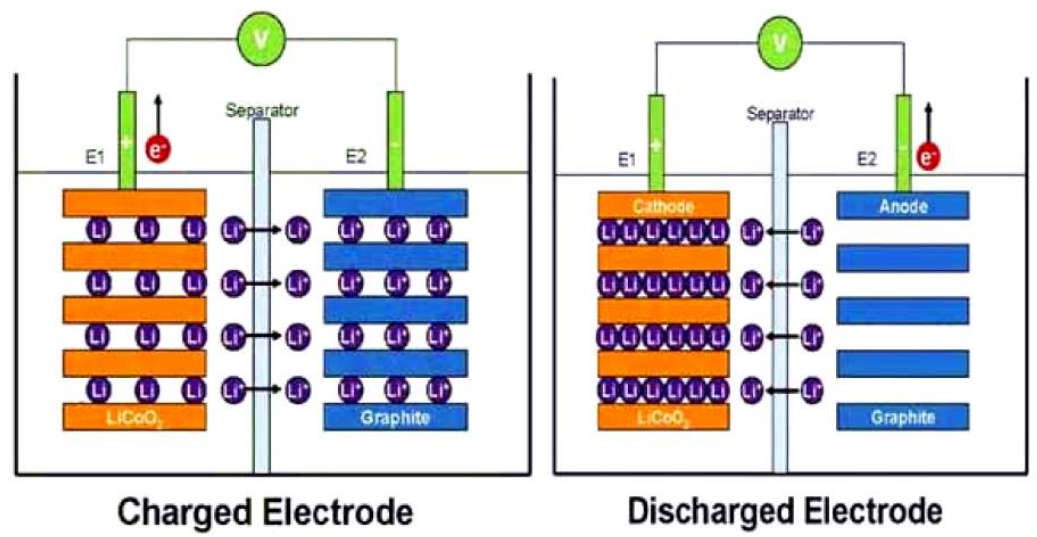

Fig 2: Working principle diagram of lithium ion battery

\section{Simulation analysis of heat generation temperature field of single cell and battery pack}

3.1 Simulation analysis of heat generation temperature field of lithium ion battery

In this paper, the lithium polymer battery produced by a company is taken as the research object, and the main technical parameters are shown in Table 1.

Table 1 Main technical parameters of lithium polymer battery

\begin{tabular}{|c|c|c|}
\hline Name & Company & Parameter \\
\hline Nominal capacity & Ah & 300 \\
\hline Nominal voltage & $\mathrm{V}$ & 3.2 \\
\hline Cut off voltage of discharge point & $\mathrm{V}$ & 2.45 \\
\hline Charge cut off voltage & $\mathrm{V}$ & 3.8 \\
\hline Operating temperature range & ${ }^{\circ} \mathrm{C}$ & $-40-70$ \\
\hline
\end{tabular}

Gambit is used to build a three-dimensional model of lithium ion battery. The length, width and height of the battery are $150 \mathrm{~mm}, 50 \mathrm{~mm}$ and $90 \mathrm{~mm}$ respectively. In order to simplify the model, the positive and negative poles are ignored in the three-dimensional model. Because the model is relatively simple, in order to improve the calculation speed and convergence speed, the model is divided into hexahedron structured grids. The grid node gap is set to 0.5 , the number of grids is set to 5,400,000, the battery shell boundary condition is set to WALL, and the heat conducting medium is set to SOLID. 
3.2 Simulation and analysis of heat generation temperature field of lithium ion battery cells

The initial temperature is set at $300 \mathrm{~K}$, the convective heat transfer coefficient under natural conditions is set at $5 \mathrm{~W} /\left(\mathrm{m}^{2} . \mathrm{K}\right)$, and the thickness of the battery shell is set at $0.3 \mathrm{~mm}$. When discharging at constant current of $100 \mathrm{~A}$, $150 \mathrm{~A}, 200 \mathrm{~A}, 250 \mathrm{~A}$ and $300 \mathrm{~A}$, one heat source is applied respectively, with the size of $3037 \mathrm{~W} / \mathrm{m}^{3}, 5444 \mathrm{~W} / \mathrm{m}^{3}$, $8444 \mathrm{~W} / \mathrm{m}^{3}, 12037 \mathrm{~W} / \mathrm{m}^{3}$ and $16222 \mathrm{~W} / \mathrm{m}^{3}$. The material of battery shell and internal material are set according to table 2. The model of this simulation is the heat generation model of lithium-ion battery. The solution used is steady flow. The selection of calculation method is shown in Figure 3. In the residual monitoring dialog box, set the convergence accuracy of the energy equation to $10^{-6}$, and the convergence accuracy of other equations to $10^{-}$ ${ }^{3}$. After iterative calculation, it will converge in step 6 .

Table 2 Physical parameters of battery shell and internal material

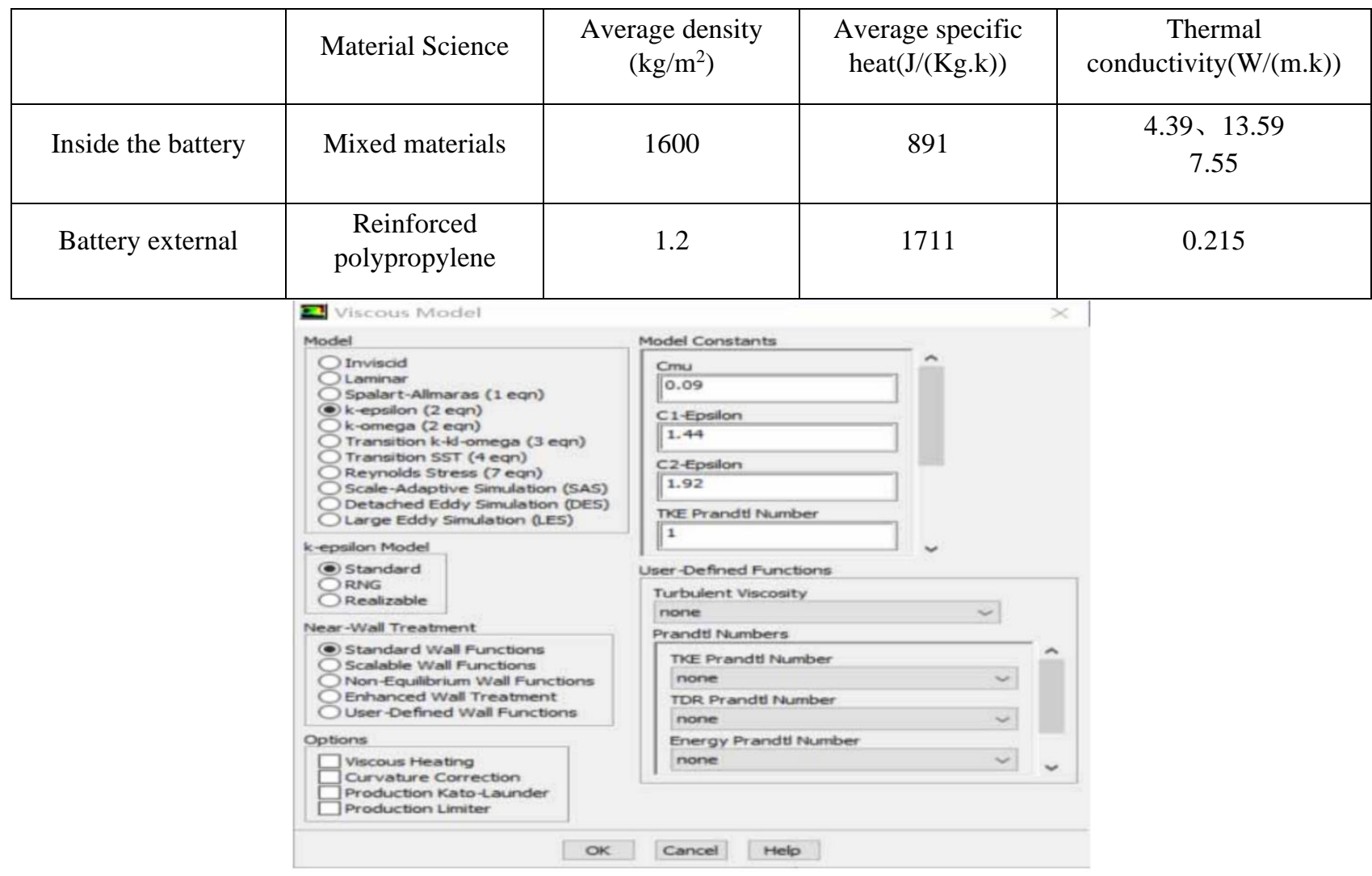

Fig 3: The choice of solving controller mode

3.3 Simulation results analysis of lithium ion battery cell

From the simulation results of heat generation temperature field distribution of $\mathrm{Li}$ ion polymer battery under different constant current discharges of 100A, 150A, 200A, 250A and 300A, it can be seen that the minimum temperature, maximum temperature and maximum temperature difference of the battery increase with the gradual increase of discharge current. The main reason is that with the increase of discharge current and discharge depth, the SOC of the battery decreases, the internal resistance of the battery increases, and the internal heat also increases, so the temperature and temperature difference increase. The constant current discharge temperature of $300 \mathrm{~A}$ single $\mathrm{Li}$ ion polymer power battery is $98^{\circ} \mathrm{C}$, which is beyond the safe temperature range of the battery, so it is necessary to heat the battery or control the discharge current in a reasonable range. When the battery cells are connected in series to form a battery pack, the temperature difference will be greater, which is extremely unfavorable for the use and maintenance of the electric vehicle battery, and the driving conditions of the vehicle are complex, sometimes requiring high-power discharge. If the cooling can not be done in time, the problem of temperature rise will have a great impact on the life of the battery and the safety of the car, and even endanger the safety of the driver. 


\section{Simulation analysis of heat dissipation temperature field of lithium ion battery pack}

\subsection{Simulation results and analysis of heat dissipation temperature field of battery pack}

The specific heat source setting and material properties setting and other settings of this simulation are consistent with the previous simulation of the battery pack. Because each simulation takes a long time, this paper only chooses the simulation of heat dissipation temperature field of battery pack in 100A constant current discharge state, and sets the air velocity of air inlet as $2 \mathrm{~m} / \mathrm{s}$ and $5 \mathrm{~m} / \mathrm{s}$ respectively for analysis. When $2 \mathrm{~m} / \mathrm{S}$ is used for ventilation and heat dissipation, the residual curve is shown in Figure 4, and converges after 134 steps.
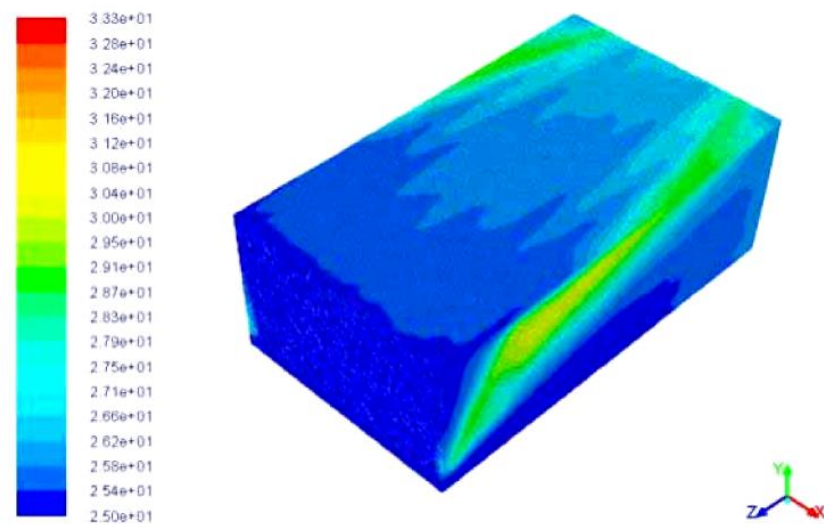

Fig 4: Residual curve of convergence for $2 \mathrm{~m} / \mathrm{s}$ ventilation and heat dissipation

The maximum temperature of the battery pack is $33.3{ }^{\circ} \mathrm{C}$, and the minimum temperature is $25^{\circ} \mathrm{C}$, which is the set initial temperature. Compared with the battery pack, the maximum temperature and minimum temperature of heat generation are lower, which shows that it has the effect of heat dissipation. The temperature distribution of the battery box can be seen that the temperature of the battery box gradually decreases from left to right. This is because the air inlet is at the lower left of the battery box, and the incoming air will bring the heat of the battery pack to the air outlet inside the battery box, while the air outlet of the battery box can not bring the heat out at once, so the temperature on the left side of the battery box is lower, while the temperature on the right side of the battery box is higher. It can also be seen that the temperature of the upper surface of the battery increases gradually from left to right, while the temperature near the air outlet decreases.

When the initial temperature is $25^{\circ} \mathrm{C}$ and the battery pack is discharging at constant current of $100 \mathrm{~A}$, compared with the temperature field of the battery pack without ventilation and heat dissipation, at the speed of $2 \mathrm{~m} / \mathrm{s}$ and $5 \mathrm{~m} / \mathrm{s}$, we can know that the maximum temperature of the battery pack decreases under the ventilation condition, and the greater the ventilation speed is. The better the cooling effect is, the lowest temperature has reached the set normal temperature, so that the battery is in a reasonable working environment, which has a good impact on the service life of the battery, will also improve the electrochemical performance of the battery, and also have a positive impact on the safety performance of the car. The specific comparison data are shown in Table 3.

Table 3 Comparison of temperature field data of battery pack under $2 \mathrm{~m} / \mathrm{s}$ and $5 \mathrm{~m} / \mathrm{s}$ ventilation conditions

\begin{tabular}{|c|c|c|c|}
\hline Discharge current $(\mathrm{A})$ & Wind speed $(\mathrm{m} / \mathrm{s})$ & Maximum temperature $\left({ }^{\circ} \mathrm{C}\right)$ & Minimum temperature $\left({ }^{\circ} \mathrm{C}\right)$ \\
\hline 100 & 0 & 37.7 & 32.8 \\
\hline 100 & 2 & 33.3 & 25 \\
\hline 100 & 5 & 31.5 & 25 \\
\hline
\end{tabular}

4.2 Simulation results and analysis of improved heat dissipation temperature field 
Because the air inlet and air outlet of the battery box designed in front mainly cool the bottom of the battery pack, but the cooling effect of the upper part is not very obvious, which will lead to high temperature in the upper part of the battery box, low temperature in the lower part, uneven temperature distribution, and also affect the life and electrochemical performance of the battery, so the air inlet and air outlet of the battery box need to be improved.

Generally, the air inlet and air outlet of the battery box are designed in two forms: serial ventilation and parallel ventilation. Serial ventilation is generally from one side of the vertical battery surface ventilation, and then from the opposite side outflow, which will lead to lower temperature at the air inlet and higher temperature at the air outlet, thus forming a larger temperature difference, which has no good effect on the heat dissipation of the battery pack. Parallel ventilation means that according to the pressure difference between the air inlet and the air outlet, the cooling air entering the air inlet automatically distributes the amount of air entering the air passage between the battery cells. Low temperature air flows into the air passage between the battery cells from the air inlet to absorb heat, and then flows out from the air outlet with heat. The design method of parallel ventilation can effectively distribute the air flow, so that the heat of each battery cell can be discharged, so as to keep the temperature of each battery cell in the battery box consistent, which has a good heat dissipation effect. The serial ventilation mode is shown in Figure 5, and the parallel ventilation mode is shown in Figure 6.

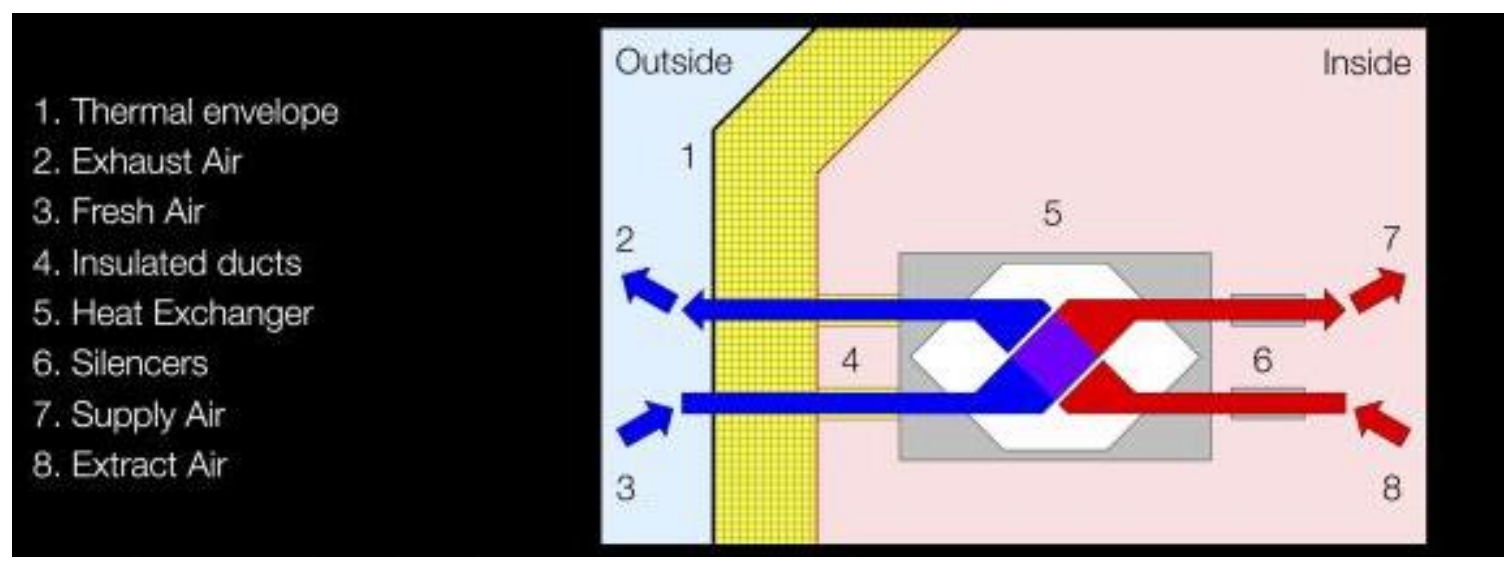

Fig 5: Serial ventilation

As shown in Figure 5, in the mode of serial ventilation, from the beginning, air enters the battery box through the air inlet, and then air flows out from the air outlet, each battery in the battery box experiences the same amount of air. However, the temperature of the cooling air is constantly getting higher in the process of flowing from the air inlet to the air outlet. When the temperature difference between the cooling air and the battery behind is not big, the cooling effect on the battery behind can hardly be achieved, so the cooling effect of the air inlet is better than that of the air outlet.

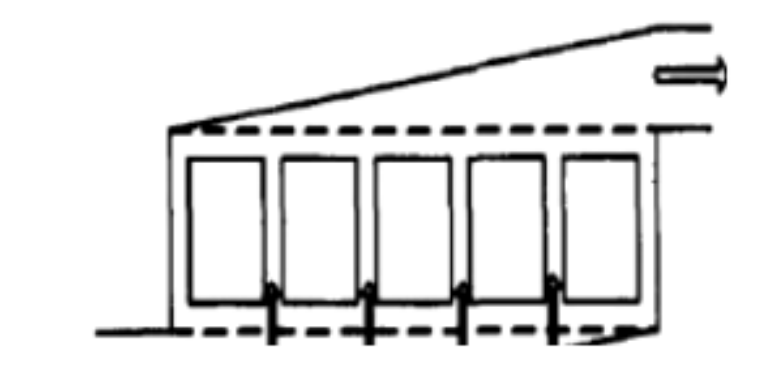

Fig 6: Parallel ventilation

As shown in Figure 6, the principle of parallel ventilation is that a total air flow enters the air inlet, and then it is divided into multiple air flows according to the number of flow channels between individual units. The flow rate 
of the multiple air streams is the same, and the flow rate mainly depends on the pressure difference. The multiple air streams enter into the flow channel between the battery cells to absorb heat and bring it out from the air outlet. The main advantage is that the multiple air streams can take away the heat of each battery cell, which can ensure the consistency of the temperature field distribution of the battery pack to a certain extent, and can play a better cooling effect.

The difference between serial ventilation and parallel ventilation is analyzed comprehensively, and it is concluded that parallel ventilation can play a more effective cooling effect on the battery pack, so this improvement uses the principle of parallel ventilation for reference. The improved battery box is shown in Figure 7, the air inlet is in the front lower part, and the air outlet is in the rear upper part.
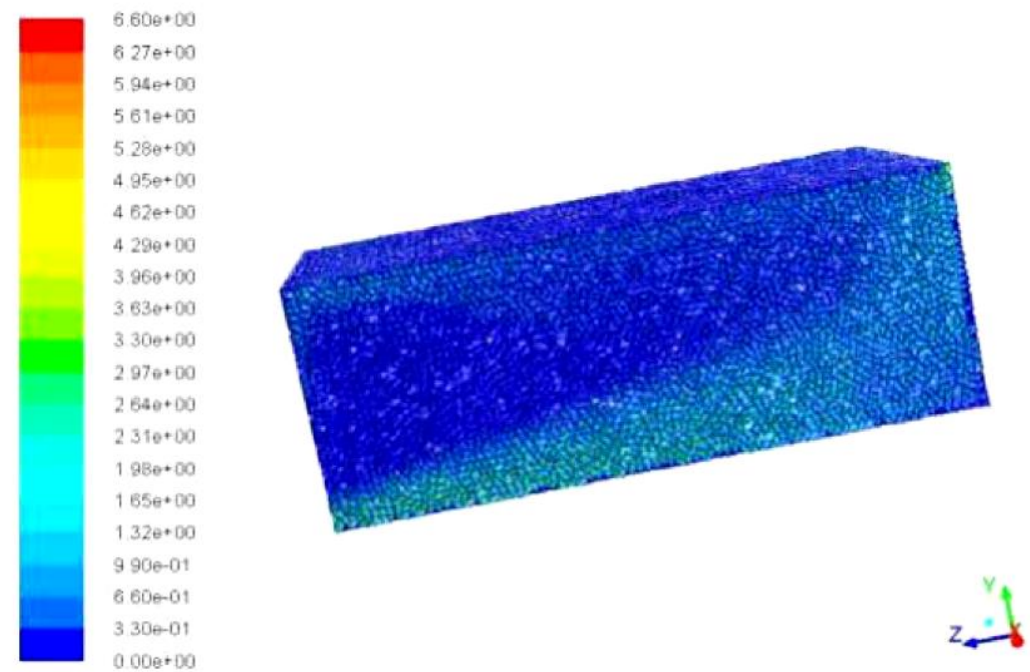

Fig 7: Improved battery box diagram

\section{Conclusion}

With the progress of pure electric vehicle technology, the promotion of state subsidies, and the improvement of infrastructure, electric vehicles have entered more and more families, and the battery thermal management system is related to the battery life and vehicle safety. Therefore, the battery thermal management system is a very important part of pure electric vehicles, which should be concerned by the relevant departments and major automobile manufacturers. In addition, the battery thermal management system should be further studied and developed. This paper takes the lithium battery of pure electric vehicle as the research object, first introduces the structure and working principle of lithium battery, and then understands the heat generation mechanism and heat transfer characteristics of lithium battery through theoretical analysis, then establishes the heat generation model of battery unit and battery pack, carries out the simulation analysis of heat generation temperature field and the simulation analysis of heat dissipation temperature field of battery pack. According to the simulation results, the air inlet and air outlet of the battery box are improved. Finally, the temperature of the working environment of the battery pack is further controlled by balancing the terminal voltage of the battery cell in the battery management system.

\section{References}

[1] Zhang Chengning, Lei Zhiguo, Dong Yugang. Research on Low Temperature Heating Method of Electric Vehicle Lithium Ion Battery . Journal of Beijing University of Technology, 2012 (09): 921925

[2] Peng Ying, Huang Rui, Yu Xiaoli. Comparative Study on Cooling Schemes of Lithium Ion Power Batteries for Electric Vehicles . Mechanical and Electrical Engineering, 2015, 032 (004): 537-543

[3] Huang Jie. Research on Lithium Battery Management System for Pure Electric Vehicles . Sci Tech 
Information, 2012, 000 (012): 4-5

[4] Ding Yi, Sun Zijian. Research on Temperature Control Performance of Electric Vehicle Power Battery Thermal Management System . Mechanical Manufacturing and Automation, 2020, V.49; No.271 (06): $192-194+202$

[5] Zhou Daohui, Yuan Dewei, Xin Ming. Research on Low Temperature Heating Method for Power Battery of Pure Electric Vehicle . Automotive Electrical Appliances, 2018, 000 (011): 15-17

[6] Zhang Jun, Zhang Lianda, Hu Chunjiao. Research on Heating and Control Strategy of Pure Electric Vehicle Battery Pack . Power Technology, 2016, 40 (009): 1845-1847

[7] Ye Kewei, Xiang Kefeng, Li Liangchao. Simulation Analysis and Research on Heat Generation Characteristics of Vda Square Power Lithium Battery . Power Technology, 2020, V.44; No.355 (04): $45-48+112$

[8] Zhang Pan. Research and Implementation of Thermal Management Technology for Electric Vehicle Power Battery . China's Strategic Emerging Industries, 2018, 000 (030): 113

[9] Zeng Jianhong, Wang Lifang, Liao Chenglin. Optimization Design of Heat Dissipation System for Lithium Ion Power Battery of Electric Vehicle . Acta Electrotechnics Sinica, 2013, 28 (1): 24-29

[10] Liu Xinlei, Cheng Zengmu. Research on Battery Matching Technology of Pure Electric Vehicle . Power Technology, 2018, 042 (003): 367-368376 